\title{
Numerical Investigation of the Influence of Span-wise Force Variation in Circular Cylinders Undergoing Vortex Induced Vibrations at High Reynolds Number
}

\author{
David Murrin*, Julio Militzer $^{\dagger}$, Neil Bose ${ }^{\ddagger}$, and Rubens Campregher ${ }^{\dagger}$ \\ * Ocean and Naval Architectural Engineering \\ Memorial University of Newfoundland, St. John's, NL A1B 3X5 \\ Email: murrin@engr.mun.ca \\ ${ }^{\dagger}$ Centre for Marine Vessel Development and Research \\ Dalhousie University, Halifax, Nova Scotia, B3J 1Z1 \\ ${ }^{\ddagger}$ Australian Maritime Hydrodynamics Research Centre (AMHRC), Newnham, Tasmania, 7248
}

\begin{abstract}
The focus of this research is on the development of a new approach for simulating vortex induced vibrations on marine risers at high Reynolds numbers. This method considers the span-wise variation of the lift and drag forces, and determines the moment acting on the cylinder. The predicted motion then consists of a rotational component to accompany the traditional cross-stream and stream-wise translations normally associated with vortex induced vibrations. This was accomplished by describing the motion of the cylinder using a set of springs and dampers. A moment acting on the cylinder causes the springs on one end to compress, and stretch on the other, thus rotating the cylinder.

A Large Eddy Simulation (LES) computational fluid dynamics code running on $163 \mathrm{Ghz}$ processors was used to calculate the unsteady flow and at each time step the hydrodynamic forces acting on the cylinder were calculated in a separate routine based on the pressure distribution around the cylinder. This information was then used to solve two second-order ordinary differential equations, which gave the velocity and displacement of the cylinder in cross-flow and rotational planes. This information was transferred back to the code where the cylinder was displaced and another cycle of calculations was started.

The simulated results showed that the correlation length was higher for a cylinder subject to pure translation compared to a cylinder free to translate and rotate in the cross-stream direction. This has implications for current numerical and experimental techniques since it has been traditionally assumed that the flow around a circular cylinder becomes two-dimensional during vortex induced vibrations. Consequently, empirical,numerical and experimental models have generally only considered cross stream and/or stream-wise translation. The extent to which the experimental apparatus or harmonic model may have influenced the behavior of the riser by eliminating span-wise amplitude variation is important information that should be considered for future riser designs.
\end{abstract}

\section{INTRODUCTION}

Vortex induced vibration is the most important dynamic response of a deep water riser [1]. The flow of seawater around these long cylinders is subject to vortex shedding. This is an unsteady oscillatory phenomenon, which causes the pressure distribution around the cylinders to fluctuate, resulting in forces perpendicular to the flow and structure. These forces excite forced oscillations of the cylinder known as vortexinduced vibrations. When the frequency of VIV approaches one of the natural frequencies of the structure, the amplitude of vibration is enhanced through a resonant phenomenon known as lock-in.

While vortex shedding on bluff bodies in the cross-stream and stream-wise directions has been described in a number of review papers over the past fifty years, the three-dimensional nature of vortex shedding has received very little attention, despite the general agreement that cylinder wakes are threedimensional for Reynolds numbers greater than 150 [2]. Moreover, the majority of three-dimensional numerical simulations have been performed at Reynolds numbers much lower than the industry relevant range of $10^{5}-10^{7}$.

The focus of this research is on the development of a new approach for simulating vortex induced vibrations on marine risers at high Reynolds numbers. This method considers the span-wise variation of the lift and drag forces, and determines the moment acting on the cylinder. The predicted motion then consists of a rotational component to accompany the traditional cross-stream translation. The movement of the cylinder, therefore, is no longer confined to a principal axis, which may influence the prescribed motion. This is accomplished by describing the motion of the cylinder using a set of springs and dampers. A moment acting on the cylinder causes the springs on one end to compress, and to stretch on the other, thus rotating the cylinder.

One of the major difficulties encountered in the simulation of this flow is the fact that the cylinder moves. In this research a number of innovative features have been incorporated into a Large Eddy Simulation (LES) computational fluid dynamics code. These features include an adaptive unstructured Cartesian grid, and a second-order Immersed Boundary (IB) Method for boundary condition specification, which allow the code to simulate flows with moving boundaries with relative 
ease. The code is used to calculate the unsteady flow and at each time step the hydrodynamic forces acting on the cylinder are calculated in a separate routine based on the pressure distribution around the cylinder. This information is then used to solve three second-order ordinary differential equations, which give the velocity and displacement of the cylinder in cross-flow, stream-wise, and rotational planes. This information is transferred back to the code where the cylinder is displaced and another cycle of calculations is started. The advantage of the Immersed Boundary Method is that there is no need to create a new mesh after the cylinder is displaced.

\section{Review of Pertinent Literature}

\section{A. Stationary Cylinders}

Reference [3] simulated the flow around a circular cylinder for $R e=5 \times 10^{5}, 1 \times 10^{6}$ and $2 \times 10^{6}$ using a Large Eddy Simulation (LES) code. They observed large, non-periodic, coherent structures in the wake of the cylinder which were different from the typical Karman vortex street found above and below critical Reynolds numbers. The simulated pressure coefficient compared well with the experimental work [4]. The predicted Strouhal number agreed well with the work of [5] for a rough cylinder, but under predicted the results of [6]. Reference [6] studied the applicability of LES for high Reynolds number flows and determined that grid-independent results for three-dimensional, time-dependent flows were a considerable challenge.

\section{B. Moving Cylinders}

Numerical simulations of vortex induced vibrations have received considerable attention in recent years, but significant, three-dimensional contributions are still very few. This is due, in part, to the incredible complexity inherent in 3-dimensional simulation of fluids at high Reynolds number, notwithstanding the fluid structure interaction that is not fully understood. Reference [7] outline four issues to be considered for any numerical simulation: modeling of the flow field, modeling of the structural vibration, modeling of the fluid-structure interaction, and data analysis. While there are a number of possible numerical methods for computational fluid dynamics, it has been shown that LES and DNS hold the most promise for understanding the wake-boundary layer interaction [8], especially at high Reynolds numbers, and for moving geometries. Limitations in computing power have prevented DNS simulations for Reynolds numbers greater than around 5000 and even these are for the simplest problems, like channel flow. It would seem then, that for the immediate future, the simulation of vortex induced vibrations can best be accomplished using large eddy simulation, and this is the focus of the work outlined in this section. Reference [9] investigated the benefits of 3D simulation using a 2D and 3D sub-grid scale LES model. They forced a cylinder to oscillate in the transverse direction at $A_{y} / D=0.11, R e=2.4 \times 10^{4}$, and $V_{r}=5.4$ (where $A_{y}$ is the amplitude of motion in the transverse direction, $D$ is the cylinder diameter, and $V_{r}$ is the reduced velocity) and compared the results. Their results showed a departure from two-dimensionality a short distance behind the cylinder that translated into more elongated and slightly weaker vortices, with a longer time averaged recirculation length. A slight phase difference between the two simulations was also observed and the $3 \mathrm{D}$ simulation showed better agreement with the experimental work of [10] at equivalent reduced velocity. Finally, the $3 \mathrm{D}$ simulation predicted smaller time averaged drag coefficient and absolute pressure than the $2 \mathrm{D}$ simulations and it was concluded that the deviations between the simulations were attributed to three-dimensionality of the wake.

A criticism of this type of simulation is the fact that it is essentially a forced oscillation, having the same limitations as the experimental equivalent. A simulation of self-excited vortex induced vibration requires a model that couples the cylinder motion and the fluid forcing function such that the two are calculated simultaneously. Reference [11] simulated self-excited VIV at $R e=8000$ for a range of damping ratios and natural frequencies using a 2D LES code and compared their results to the work of [12]. The immediate concern with a $2 \mathrm{D}$ simulation is the assumption of two-dimensionality. Most 2D codes over-predict the drag coefficient for large Reynolds numbers by $5-10 \%$ [11] and the results from 3D codes provide better agreement with experimental data (confirmed by [9]). Reference [11] argue that a 2D simulation has its merits since the lift and drag coefficients are predicted more accurately when VIV begins, because at this point, the wake correlation length increases and the $3 \mathrm{D}$ influence of vortex shedding is diminished. They do concede, however, that there is some difference in the vortex shedding behavior for turbulent flows.

Perhaps the most important result from the study was the absence of the $2 \mathrm{P}$ and $2 \mathrm{~S}$ modes of vibration identified by [13], [14] [15], and [12]. Reference [11] also concluded that a direct comparison between forced and self excited oscillations cannot be made, especially when self-excited oscillation is not sinusoidal and phase angle is not constant.

Reference [16] assessed the validity of 2-D assumptions on mean drag and flow induced forces for a stationary circular cylinder with $R e=100$ and aspect ratio of 16, using finite volume and lattice Boltzmann numerical techniques. They found that the calculated mean drag and r.m.s. lift coefficients varied greatly across the span and that vortex shedding changed from parallel to oblique over the time range of $600 \leq t \leq 900$. They also found that lift and drag signals went through a series of transitions for $t \leq 200,200 \leq t \leq 500$, and $t \geq 500$, and concluded that the variation partially explains why there is so much scatter in the lift and drag coefficients in the literature.

In 2005, [17] performed a series of VIV experiments on a hanging cylinder pin connected at one end, and free in the other. The results showed a new mode of vortex shedding near the pin in which a pair of co-rotating vortices were formed. This mode was subsequently named the $2 \mathrm{C}$ mode of vortex shedding, and illustrated that all of the shedding mode shapes for cylinders undergoing VIV have may not have been discovered. 


\section{Methodology OF ReseARCH}

The simulations were performed using in-house CFD software, known as the Numerical Wind Tunnel (NWT), initially developed at Dalhousie University and expanded at Memorial University for the purpose of this research. The NWT is a parallel processing tool used to solve three-dimensional turbulent flows around bodies of generic geometry. The simulation domains are discretized by the Finite Volume Method over unstructured anisotropic Cartesian staggered grids. Since three-dimensional numerical simulations typically require a large amount of data to be processed, the NWT has parallel processing capabilities such that the computational grid is automatically distributed among the available processors to optimize load balancing (see [18]). A multi-scale LES turbulence model was used to model the small scales of flow and prevent numerical divergence [19]. The incompressible Navier-Stokes equations in tensor conservation form can be written as:

$$
\frac{\partial u_{i}}{\partial t}+\frac{\partial\left(u_{i} u_{j}\right)}{\partial x_{j}}=-\frac{\partial p}{\partial x_{i}}+\frac{1}{R e_{\tau}} \frac{\partial^{2}\left(u_{i}\right)}{\partial x_{j} \partial x_{j}}+f_{i},
$$

where $u_{i}$ is the Cartesian velocity component, $p$ is the pressure, and $f_{i}$ is the body force term. The turbulent Reynolds number is given by $R e_{\tau}=U_{0} D / \nu_{\text {eff }}$, where $\nu_{e f f}$ is the effective kinematic viscosity, evaluated by the LES turbulence model as:

$$
\nu_{e f f}=C_{S}^{2} \Delta^{2} \sqrt{S_{i j} S_{i j}},
$$

where $S_{i j}=\frac{1}{2}\left[\frac{\partial u_{i}}{\partial x_{j}}+\frac{\partial u_{j}}{\partial x_{i}}\right]$, and $C_{S}$ is the Smagorinsky constant. The filter size $\Delta$ was evaluated considering the dimensions of the current and adjacent cells. The NWT solves the pressure-velocity coupling by a fully implicit FractionalStep method. The linear system generated by the discretization of the momentum equation is solved by a Gauss-Seidel algorithm and the Poisson equation for the pressure correction field is solved by the Conjugate Gradient algorithm.

\section{A. Lift and Drag Forces}

For cylindrical surfaces at moderate Reynolds numbers it has been shown that the contribution to the lift and drag forces from shear stresses is minimal [20]. For this work, the drag and lift forces were pressure dominated and the contribution of shear stress was therefore ignored. The resultant of the pressure distribution was obtained by integrating the pressure values over the cylinder surface, with the lift force on the cylinder given by:

$$
F_{l}(t)=\int p \sin \phi d A
$$

and the lift coefficient:

$$
C_{l}=\frac{2 F_{L}(t)}{\rho V_{0}^{2} A} .
$$

Similarly, the drag force and drag coefficient on a cylinder are given by (5) and (6), respectively:

$$
F_{d}(t)=\int p \cos \phi d A
$$

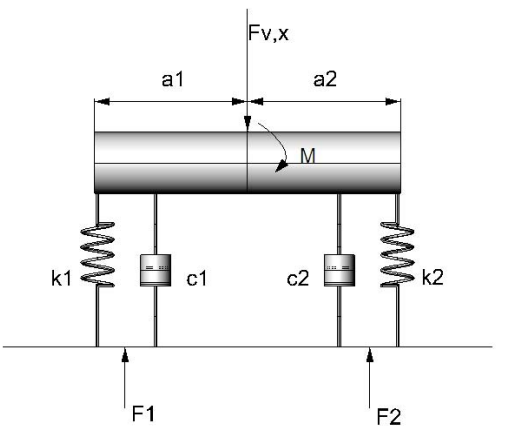

Fig. 1. Arbitrary two degree of freedom spring-mass-damper system

$$
C_{d}=\frac{2 F_{D}(t)}{\rho V_{0}^{2} A}
$$

where $p$ represents the pressure contribution and $F_{d}$ and $F_{l}$ represent the forces of drag and lift, respectively. The fluid density, angle relative to the front stagnation point, free stream velocity and characteristic area were given by $\rho, \phi, V_{0}$, and $A$, respectively.

\section{B. Accounting for the span-wise variation in forces along the cylinder span.}

In order to account for the force variation along the span of a cylinder, a rotational component was introduced into the system of VIV equations traditionally used to model translation. The movement of the cylinder, therefore, is no longer confined to the principal axis, which may influence the prescribed motion.

For an arbitrary two-d.o.f. spring-mass-damper system, as shown in Figure 1, the coupled equations of motion are defined by

$$
\begin{gathered}
m \ddot{x}=F_{v}-F_{1}-F_{2} \\
I \ddot{\theta}=M_{v}+F_{1} a_{1}-F_{2} a_{2},
\end{gathered}
$$

where $F_{v}$ and $M_{v}$ are the external force and moment on the system, while $F_{1}$ and $F_{2}$ are the interactive forces between the two d.o.f. spring-mass-damper system and $a_{1}, a_{2}$ are the distances shown in Figure 1 .

In general, the equation of motion for a single spring-massdamper can be written as:

$$
m \ddot{y}+c \dot{y}+k y=F(t) .
$$

For the purposes of VIV, the following non-dimensional terms are introduced

$$
\begin{gathered}
\zeta=\frac{c}{2 \sqrt{k m}} \\
\frac{k}{m}=\left(2 \pi f_{n}\right)^{2} \\
U^{*}=\frac{U_{\infty}}{f_{n} D}
\end{gathered}
$$




$$
\begin{gathered}
m^{*}=\frac{m}{\left(\frac{\pi}{4} \rho D^{2}\right)} \\
C_{L}(t)=\frac{2 F_{L}(t)}{\rho U_{\infty}^{2} A},
\end{gathered}
$$

where $\zeta$ is the is the material damping ratio, $k$ and $c$ are the spring and damper constants, respectively, and $f_{n}$ is the natural frequency. The free-stream velocity, cylinder diameter, and reduced velocity are given by $U_{\infty}, D$, and $U^{*}$, respectively. The ratio of the cylinder mass $(m)$ to the displaced fluid mass $\left(m_{d}=\frac{\pi}{4} \rho D^{2}\right)$ is known as the mass ratio, $m^{*}$.

Substituting (10) through (14) into (9) and simplifying gives:

$$
\ddot{y}+\left(\frac{4 \pi \zeta}{U^{*}}\right) \dot{y}+\left(\frac{2 \pi}{U^{*}}\right)^{2} y=\frac{2}{\pi} \frac{C_{L}(t)}{m^{*}},
$$

which is recognized as a suitable equation for describing the cross-stream response of a structure to vortex induced vibrations. This equation may be written compactly as:

$$
\ddot{y}+c_{1} \dot{y}+k_{1} y=\frac{2 C_{L}(t)}{\pi m^{*}},
$$

where $c_{1}=\left(\frac{4 \pi \zeta}{U^{*}}\right)$ and $k_{1}=\left(\frac{2 \pi}{U^{*}}\right)^{2}$.

For multiple spring-mass-damper systems, (16) can be written as:

$$
m \ddot{y}+\sum c_{i} \sum \dot{y}_{i}+k_{i} y_{i}=F
$$

and equivalently,

$$
\ddot{I} \theta-\sum c_{i} \dot{y}_{i} a_{i}-\sum k_{i} y_{i} a_{i}=M .
$$

Returning to Figure 1, the free body diagram is shown in Figure 2 and the equations of motion for the cross-stream and rotational directions are:

$$
\begin{gathered}
m \ddot{y}+c_{1} \dot{y_{1}}+k_{1} y_{1}+c_{2} \dot{y_{2}}+k_{2} y_{2}=F \\
I \ddot{\theta}-c_{1} \dot{y_{1}} a_{1}-k_{1} y_{1} a_{1}+c_{2} \dot{y_{2}} a_{2}+k_{2} y_{2} a_{2}=M .
\end{gathered}
$$

Substituting $y_{1}=y-a_{1} \sin \theta$ and $y_{2}=y+a_{2} \sin \theta$ and assuming small amplitudes of oscillation $(\sin \theta \simeq \theta),(19)$ and (20) may be written as

$$
\begin{gathered}
m \ddot{y}+c_{1}\left(\dot{y}-a_{1} \dot{\theta}\right)+k_{1}\left(\dot{y}-a_{1} \theta\right)+ \\
c_{2}\left(\dot{y}+a_{2} \dot{\theta}\right)+k_{2}\left(\dot{y}+a_{2} \theta\right)=F \\
I \ddot{\theta}-c_{1}\left(\dot{y}-a_{1} \dot{\theta}\right) a_{1}-k_{1}\left(\dot{y}-a_{1} \theta\right) a_{1}+ \\
c_{2}\left(\dot{y}+a_{2} \dot{\theta}\right) a_{2}+k_{2}\left(\dot{y}+a_{2} \theta\right) a_{2}=M .
\end{gathered}
$$

Equation (22) may be uncoupled by noting that $k_{1} a_{1}=$ $k_{2} a_{2}$ and $c_{1} a_{1}=c_{2} a_{2}$ (i.e. $a=$ Length $/ 2$ ):

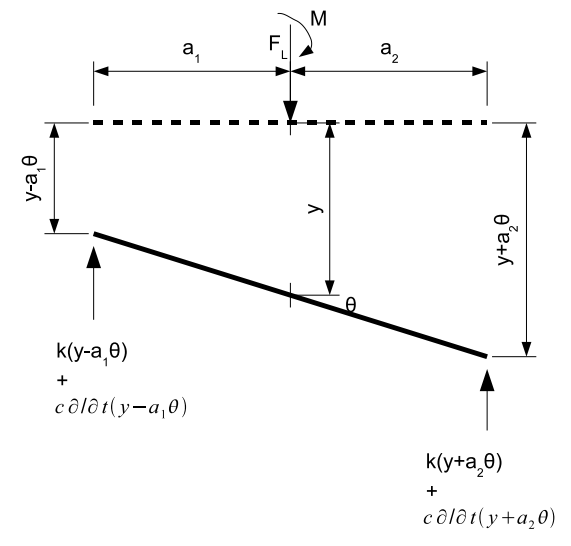

Fig. 2. Free body diagram for multiple spring-mass-damper system

$$
\begin{gathered}
m \ddot{y}+2 c \dot{y}-2 k y=F \\
I \ddot{\theta}+\left(c_{1}+c_{2}\right) a^{2} \dot{\theta}+\left(k_{1}+k_{2}\right) a^{2} \theta=M .
\end{gathered}
$$

This approach uses two springs and two dampers, each having coefficients that are half as large as the coefficients traditionally applied to risers. In terms of the traditional coefficients, (23) and (24) can then be written as:

$$
\begin{gathered}
m \ddot{y}+c \dot{y}-k y=F \\
I \ddot{\theta}+c a^{2} \dot{\theta}+k a^{2} \theta=M .
\end{gathered}
$$

Noting that (25) and (16) are equivalent, (26) is expanded by replacing the constants $c$ and $k$ with the well-known riser parameters and substituting the equation for the moment of inertia of a cylinder rotated about its cross-sectional axis:

$$
I=\left(\frac{m r^{2}}{4}+\frac{m L^{2}}{12}\right) .
$$

For most cases $L^{2} \gg r^{2}$, and (27) can be simplified to

$$
I \approx \frac{m L^{2}}{12}
$$

In a manner analogous to the mass ratio, the inertia ratio is defined as the ratio of the body inertia to the inertia of the displaced fluid, and is given by:

$$
I^{*}=\frac{I}{I_{d}} .
$$

The inertia term can therefore be replaced by

$$
I=\frac{m_{d} L^{2}}{12} I^{*}
$$

The moment can also be nondimensionalized by the moment coefficient, $C_{m}$ :

$$
M=\left(\frac{1}{2} \rho V_{\infty}^{2} L^{2}\right) C_{m}
$$




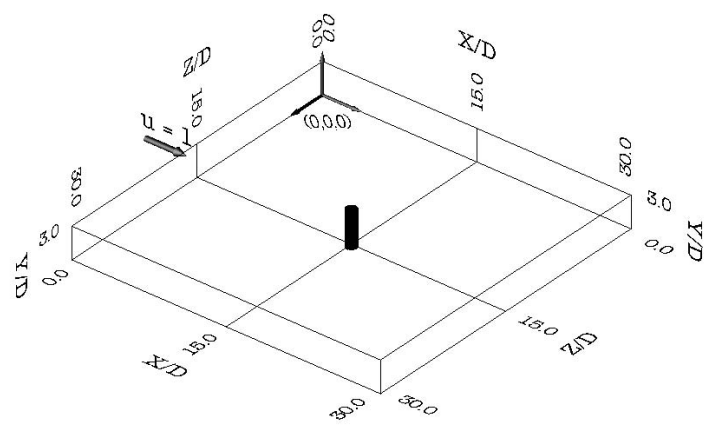

Fig. 3. Calculation domain

The rotational response of a structure to vortex induced vibrations is obtained by substituting (30), (31) and the nondimensional terms previously described into (26) and simplifying:

$$
\ddot{\theta}+\frac{12\left(\pi \zeta f_{n}\right) m^{*}}{I^{*}} \dot{\theta}+\frac{12\left(\pi f_{n}\right)^{2} m^{*}}{I^{*}} \theta=\frac{24}{\pi I^{*}} C_{m} .
$$

Together with the rotational response given by (32), the expression for the cross-stream response is given by the following equation:

$$
\ddot{y}+\left(\frac{4 \pi \zeta}{U^{*}}\right) \dot{y}+\left(\frac{2 \pi}{U^{*}}\right)^{2} y=\frac{2}{\pi} \frac{C_{L}(t)}{m^{*}} .
$$

These equations were solved at each time-step using 4th order Runge-Kutta routines. The location of the immersed boundary was then updated with the resultant displacements and the Cartesian mesh was refined to reflect the new location of the boundary. The LES then continued and the predicted lift and drag forces were sent to the Runge-Kutta routines and the cycle continued until the target number of timesteps was reached.

\section{RESUlts AT $R e=10^{5}$}

The calculation domain for each of the simulations, shown in Figure 3, was set to 30 cylinder diameters $(30 D)$ in the streamwise, $X$-direction, and $30 D$ in the cross-stream, $Z$ direction. The span-wise length was set to be $3 D$, sufficiently large to capture significantly large three-dimensional spanwise wake effects [21]. The cylinder was located at the centre of the $X$ and $Z$ planes, and it's axis spanned the extent of the domain in the $Y$ direction. Periodic boundary conditions were imposed in the span-wise direction, and slip boundaries were imposed in the cross-stream direction. A uniform inlet velocity of $1 \mathrm{~m} / \mathrm{s}$ was specified at the inlet and the downstream boundary was prescribed by a convective outlet. Furthermore, the simulations were each performed using $163 \mathrm{GHz}$ processors in parallel.

The blockage ratio can be defined as the ratio of the cylinder diameter to the height of the test section in the cross-stream direction and the blockage effect is said to be negligible if this ratio is less than $3-5 \%$. Reference [22] has shown that larger blockage ratios tend to increase the separation angle and drag coefficient, and decrease the size of vortices. For this reason, all of the simulations in this work maintained a blockage ratio below $5 \%$.

\section{A. Grid Independence}

The computational expense of three-dimensional simulations at high Reynolds numbers is extremely (and sometimes prohibitively) high, due to the level of mesh refinement required. This, coupled with limited computer resources and time restrictions did not allow for a traditional grid independence study to be performed for these simulations. A new approach was therefore required to establish the grid sensitivity. It was decided to perform a grid independence study over three grids that were only one cell thick in the span-wise direction (essentially, a two-dimensional grid), but otherwise identical to the domain shown in Figure 3. Rather than tracking changes in the results against the number of cells in the mesh, the grid adaptation criterion was chosen as the dependent variable in the grid refinement study. This parameter specifies the tolerance for which the error across a given cell calls the automatic mesh refinement routine. The mesh density may be controlled by adjusting the value of the the grid adaptation criterion and a grid refinement study can therefore by undertaken by adjusting its value to produce coarse and dense meshes. Assuming that velocity fluctuations in the flow-field are largest in the wake of the cylinder, the domain in Figure 3 should also be grid independent for the same value of the grid adaptation criterion.

Three different grids (and hence grid adaptation criteria) were used to obtain grid independent results. Each simulation was started from rest and carried out for 30 dimensionless time-steps in order for the wake and vortex street to become well established. The average values of $C_{d}$ and root-meansquare of $C_{l}$ were calculated as well as the Strouhal number. The grid sensitivity study showed that the simulations approached grid independent results for these parameters using an adaptation criteria of $4 E-05$. The domain shown in Figure 3 was therefore assumed to be grid independent for an adaptation criterion of 4E-05. Figures 4 and 5 show the mesh near the cylinder. This mesh, consisting of roughly one million cells, has been automatically refined according to the grid independence study, and shows that the grid density is greater in the wake of the cylinder than along the span. This means that assuming the refinement criterion was sufficient in the wake (as determined by the grid independence study), the variation in the span-wise direction was sufficiently refined as well.

\section{B. Stationary Cylinder}

Figure 6 shows two cycles of the lift coefficient, along with the drag coefficient for this flow. The predicted r.m.s lift coefficient for the stationary cylinder was computed to be $C_{l, r m s}=0.54$, which is within $2 \%$ of the experimental work of [23] with $C_{l, r m s}=0.55$. The average drag coefficient for the stationary cylinder was $C_{d}=1.15$, which is within $4 \%$ of the experimental measurements of [24] and [6], who obtained time averaged drag coefficients of $C_{d}=1.20$. Furthermore, 


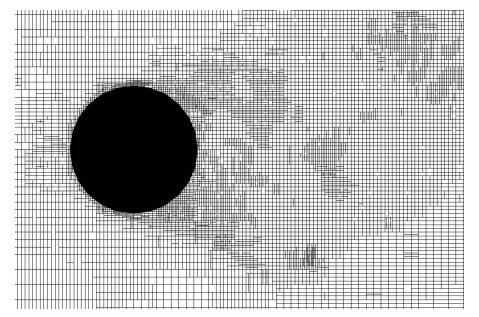

Fig. 4. Refined mesh around cylinder for flow past stationary cylinder at $R e=10^{5}$

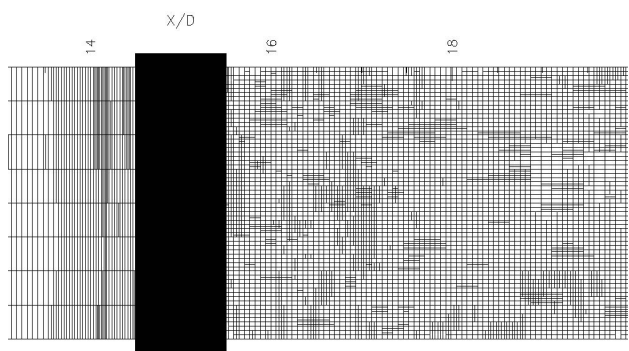

Fig. 5. Refined mesh along span for flow past stationary cylinder at $R e=$ $10^{5}$

the predicted Strouhal number of $S t=0.183$ is also within $4 \%$ of the simulated results of [21] with $S t=0.190$, and $2 \%$ of empirical result of $S t=0.186$ obtained by [25]. The inset of Figure 6 shows the amplitude spectrum of lift, and confirms this value with a clear resonant peak at $S t=0.183$. Plots of span-wise vorticity at three different locations along the span of the cylinder are shown in Figures 7,8 and 9. The vortex shedding pattern is such that in a cycle two vortices are released, one from the bottom and another from the top. This shedding behavior can then be identified as $2 \mathrm{~S}$, namely two single vortices. Although the shedding pattern remains $2 \mathrm{~S}$ along the span of the cylinder, the nature and timing of shed vortices varies considerably. This can perhaps be seen most effectively in the three-dimensional iso-surface plot of span-wise vorticity shown in Figure 10. It is clear from the figure that vortices generated inside the riser boundary layer are shed downstream and form coherent structures in the $2 \mathrm{~S}$ pattern. The pattern persists along the span and an increasingly

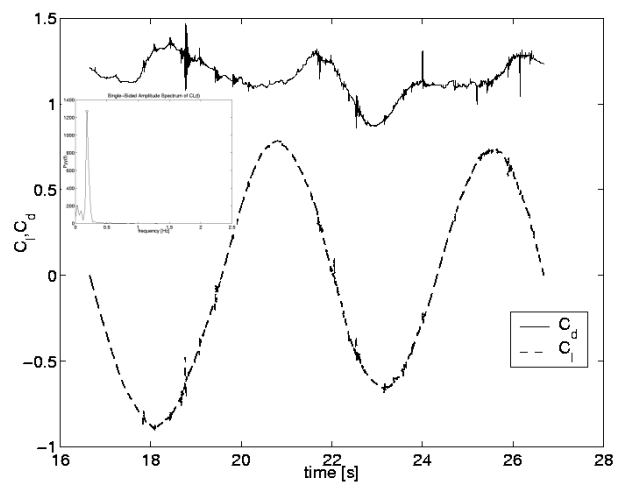

Fig. 6. Coefficients of lift and drag for stationary cylinder at $R e=10^{5}$

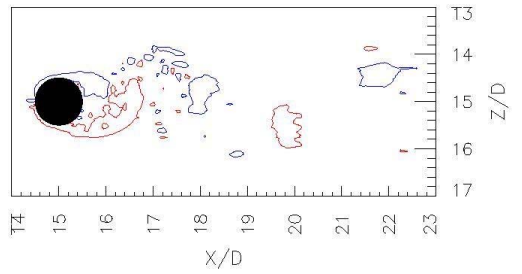

Fig. 7. Vorticity contours for stationary cylinder at $R_{e}=10^{5}$, and y $=0.5$

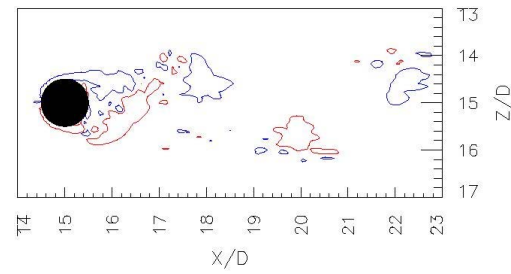

Fig. 8. Vorticity contours for stationary cylinder at $R_{e}=10^{5}$, and y $=1.5$

three-dimensional flow field develops downstream. The flow is not uniform along the span of the cylinder, which indicates that the flow cannot be assumed to be two-dimensional. An effective way of quantifying the degree of three-dimensionality in a flow-field is by examining the spatial correlation along the span. The correlation coefficient, $\gamma_{i j}$, indicates the strength and direction of a linear relationship between two random variables, and is given by [16]:

$$
\gamma_{i j(\tau)}=\frac{\sum_{t}\left\{\left[C_{l}\left(Y_{i}, t+\tau\right)\right]\left[C_{l}\left(Y_{j}, t\right)\right]\right\}}{\sqrt{\sum_{t}\left[C_{l}\left(Y_{i}, t+\tau\right)\right]^{2}} \sqrt{\sum_{t}\left[C_{l}\left(Y_{j}, t\right)\right]^{2}}},
$$

where $Y_{i}$ represents the spatial position along the span and $\tau$ represents the time delay between samples. In general terms, correlation refers to the departure of two variables from independence, and a value of $\gamma_{i j}$ equal to unity indicates that the variables are perfectly correlated. The coefficient $\gamma_{i j}$ between the mid-span $(Y=0)$ and another location along the span at zero time delay $(\tau=0)$ is shown in Figure 11. The figure shows that the flow is most correlated near the mid-span, and becomes less and less correlated towards the ends of the cylinder. Furthermore, the flow is shown to be anti-symmetric about the mid-span.

Another measure of the three-dimensionality of a field is the span-wise correlation length, $\Lambda$, scaled by the cylinder diameter. There exists a correlation length over which the the force fluctuations in the wake may be described as perfectly

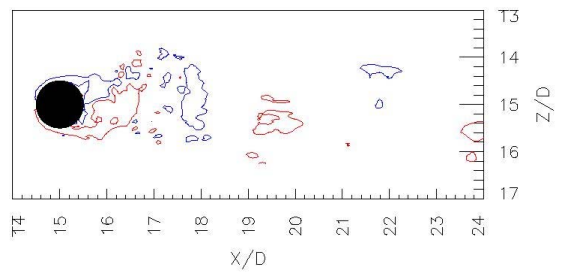

Fig. 9. Vorticity contours for stationary cylinder at $R_{e}=10^{5}$, and y $=2.5$ 


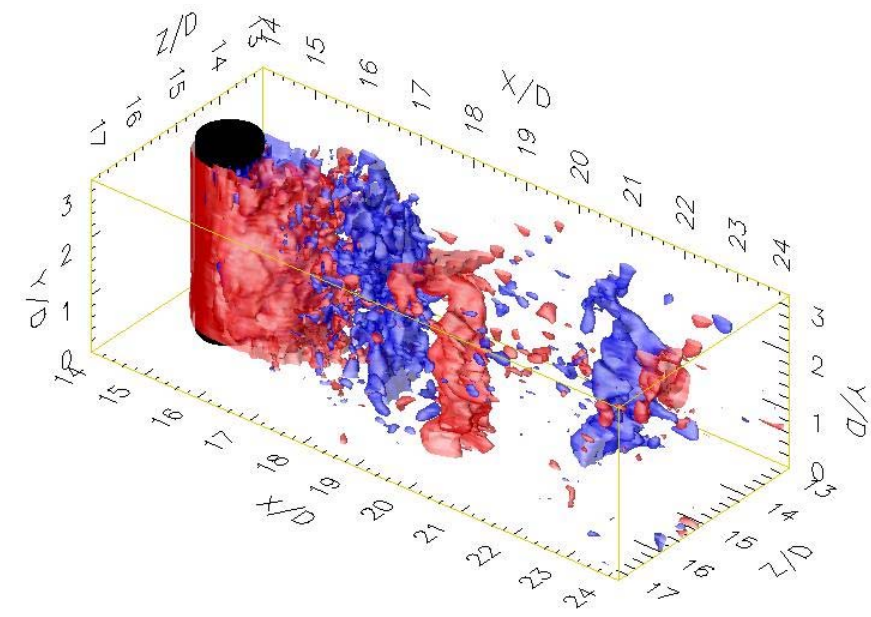

Fig. 10. Instantaneous span-wise vorticity iso-surfaces for flow past stationary cylinder at $R_{e}=10^{5}$. Iso-surfaces +2.0 and -2.0

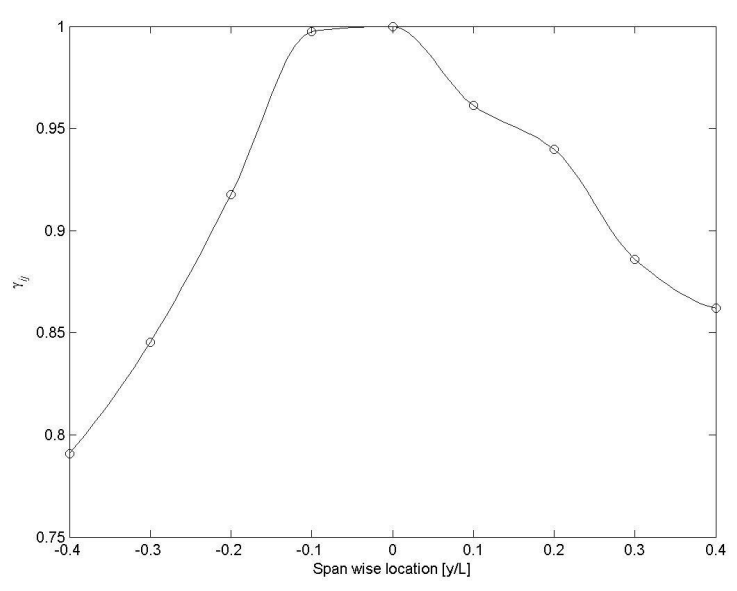

Fig. 11. Cross correlation coefficient along span of stationary cylinder at $R e=10^{5}$

correlated. Knowledge of $\Lambda$ also has significance for vortexinduced vibration modeling since many empirical models use it as a modeling parameter, and it is important to identify the span-wise computational dimension to use in 3-D CFD codes to capture significant flow features. The correlation length can be computed by integrating the correlation coefficient over the cylinder length [26]:

$$
\Lambda=\int_{-L / 2}^{L / 2} \gamma_{i j(z)} d z .
$$

The correlation length for this field was computed to be 2.7, which indicates that the force fluctuations are highly correlated for roughly $90 \%$ of the cylinder length. This value compares reasonably well with the empirical formulas presented by [25]:

$$
\Lambda=2.6\left(\frac{R e}{2.4 \times 10^{5}}\right)^{-0.2}=3.1
$$

TABLE I

VIV PARAMETERS FOR CYLINDER TRANSLATING IN CROSS-STREAM DIRECTION

\begin{tabular}{|c||c||c|}
\hline Parameter & Trans & Trans+rot \\
\hline$R e$ & $10^{5}$ & $10^{5}$ \\
\hline$U^{*}$ & 6.055 & 6.055 \\
\hline$\zeta^{*}$ & 0.005 & 0.005 \\
\hline$m^{*}$ & 2.65 & 2.65 \\
\hline$I^{*}$ & $\mathrm{n} / \mathrm{a}$ & 2.65 \\
\hline
\end{tabular}

\section{Moving CYlinder}

The periodic flow-field produced by the stationary cylinder simulation was used as a starting point in the VIV simulation, and the moment induced by the lift forces, as well as the resultant lift and drag forces on the cylinder were input into the Runge-Kutta routines described previously. The cylinder position was then updated with the cross-stream displacements and rotational angle, and the flow-field was solved by the NWT. At each subsequent time step, the moving cylinder routine was re-invoked and the LES flow simulation for the displaced cylinder was repeated until the target number of time steps was achieved. The simulated results presented in this chapter required considerable computational resources which precluded a long time statistical analysis. The results, therefore concentrate primarily on the behavior of the cylinder at the onset of vortex induced vibrations, and have been performed mainly using a 16 processor $(3.6 \mathrm{GHz})$ PC cluster located at the Centre for Marine Vessel Development and Research at Dalhousie. Towards the end of this research the Atlantic Computational Excellence Network (ACEnet) was also used to perform simulations using $16,2.6 \mathrm{GHz}$ processors. The case of a cylinder undergoing vortex induced vibrations in the crossstream direction was first investigated to determine the flow characteristics and establish a baseline for the remaining tests, which allowed the cylinder to translate and rotate in plane parallel to the cross-stream direction. The differences between the simulations, were therefore attributed to the rotation caused by a variable amplitude of vibration along the span of the cylinder.

In order to ensure that all of the moving cylinder simulations started with a well established flow field, and that each had a common set of initial conditions, all of the moving cylinder simulations presented in this chapter began at the end of the periodic regime of the stationary cylinder. Table I summarizes the VIV parameters used in these simulations.

\section{A. Cylinder Translating in Cross-stream Direction}

The simulation of vortex induced vibrations on a cylinder translating in the cross-stream direction is presented in this section in order to establish a baseline against which the effects of rotation may be measured.

For the present case of a cylinder translating in the crossstream direction, there is no rotational component, and hence 


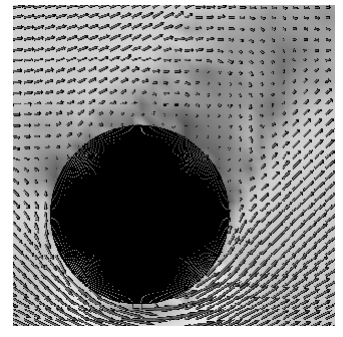

Fig. 12. Velocity vectors at $\mathrm{y}=0.5$ for cylinder at $R_{e}=10^{5}$ oscillating in stream-wise direction

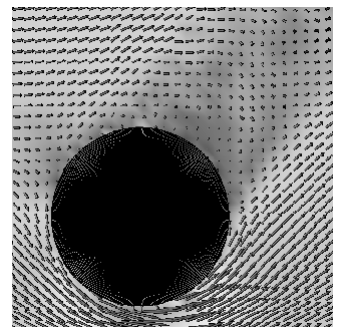

Fig. 13. Velocity vectors at $\mathrm{y}=1.5$ for cylinder at $R_{e}=10^{5}$ oscillating in stream-wise direction

$I^{*}$ is not significant. The three-dimensional characteristics of this flow near the wake of the cylinder at the mid-span and $0.5 D$ from each end are shown in the velocity vector plots in Figures 12, 13, and 14. No clear vortex shedding pattern is obvious at the onset of motion, and the figures look remarkably similar to each other along the span of the cylinder. This indicates that the wake of the cylinder is less three-dimensional than the case of the stationary cylinder. Three-dimensional iso-surfaces of velocity for this flow, presented in Figure 15, show that while there is some variation along the span of the cylinder, the flow is essentially behaving similarly along the cylinder. To quantify the degree of three-dimensionality, the correlation coefficients $\left(\gamma_{i j}\right)$ were computed, and are presented in Figure 16. Values of $\gamma_{i j}$ near unity indicate that the lift coefficient is highly correlated along the entire span of the cylinder. Compared with the results presented for the stationary cylinder, it is clear that the forces along the cylinder span become more correlated when the cylinder is allowed to move in the cross-flow direction. This is an important result, because it supports the commonly held notion that the flow becomes two-dimensional once the cylinder is allowed

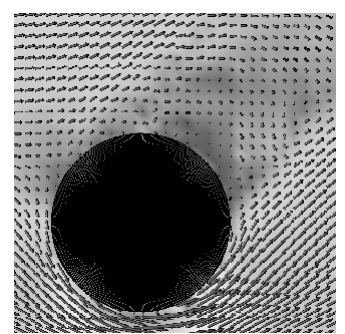

Fig. 14. Velocity vectors at $\mathrm{y}=2.5$ for cylinder at $R_{e}=10^{5}$ oscillating in stream-wise direction

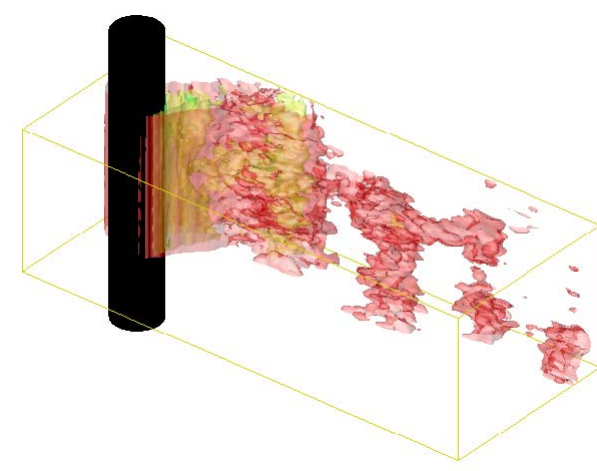

Fig. 15. Instantaneous iso-surfaces stream-wise velocity for flow past a moving cylinder. Iso-surfaces $+0.5,0.2$ and $-0.5,-0.2$

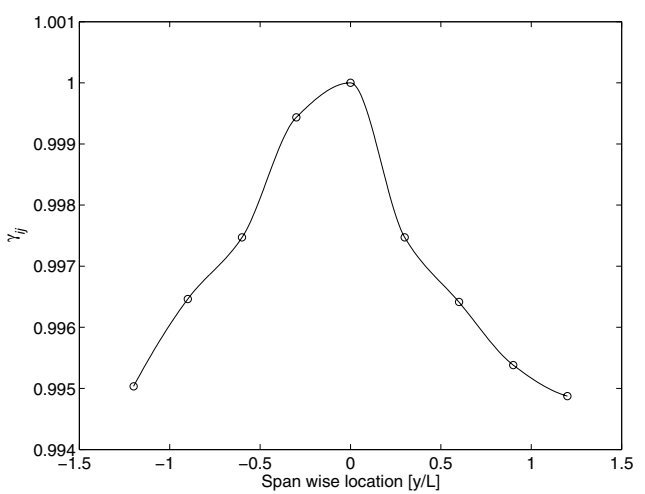

Fig. 16. Cross correlation coefficient along span of cylinder translating in cross-stream direction at $R e=10^{5}$

to respond to it's shed vortices. Perhaps the uniform motion in the direction of the force organizes the wake and governs the flow. But the question remains as to whether this behavior will continue as the cylinder is allowed to rotate and translate in the same plane.

The correlation length, $\Lambda$, for this flow-field was computed to be 2.98, which indicates that the force fluctuations in the wake are perfectly correlated for essentially the entire length of the $3 m$ cylinder. This value is higher than $\Lambda=2.7$ computed for the stationary cylinder, thus providing further evidence of two-dimensionality in the flow field once the cylinder moves.

\section{B. Cylinder Translating and Rotating in Cross-stream Direc-} tion

The simulation of vortex induced vibrations on a cylinder free to translate and rotate with it's inertia ratio equal to it's mass ratio is presented in this section. This is a realistic value of the inertia ratio for a uniform cylinder, since if the mass is uniformly distributed along the span it follows that:

$$
I^{*}=\frac{I}{I_{d}}=\frac{\left(\frac{m L^{2}}{12}\right)}{\frac{\left(m_{d} L^{2}\right)}{12}}=\frac{m}{m_{d}}=m^{*} .
$$

The three-dimensional characteristics of this flow near the wake of the cylinder at the mid-span and $0.5 \mathrm{D}$ from each end are shown in the velocity vector plots in Figures 17, 18 


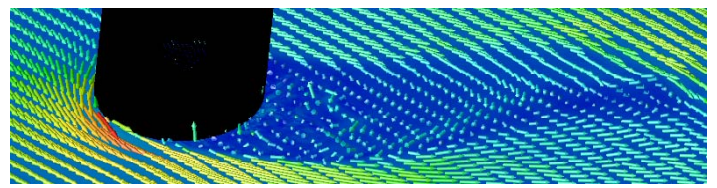

Fig. 17. Velocity vectors for cylinder at $R_{e}=10^{5}$ and $I^{*}=m^{*}$, at y $=$ 0.5

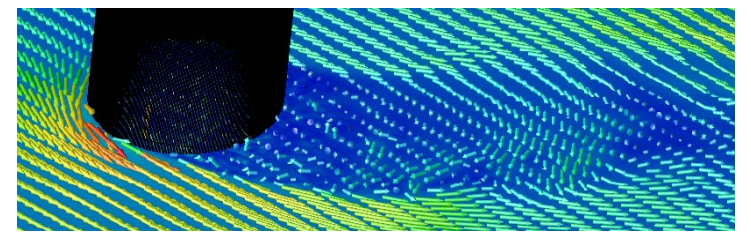

Fig. 18. Velocity vectors for cylinder at $R_{e}=10^{5}$ and $I^{*}=m^{*}$, at y $=$ 1.5

and 19. The flow pattern varies along the span, and shows a small pair of co-rotating vortices at the mid-span, and hints of it near the extremities. In the literature, this is known as the $2 \mathrm{C}$ pattern and has been identified by [17] in experiments on a cylinder pivoting about a pin connection at the top of the span at $R e \approx 1200$.

Three-dimensional iso-surfaces of velocity for this flow, presented in Figure 20, show that there is variation along the span of the cylinder.

The correlation coefficients along the span of the cylinder with $I^{*}=m^{*}$ are presented in Figure 21. There is a narrow region of highly correlated flow at the cylinder mid-span and less correlation towards the ends of the cylinder. The correlation length, $\Lambda$, for this flow-field was computed to be 2.6, which indicates that the force fluctuations in the wake are

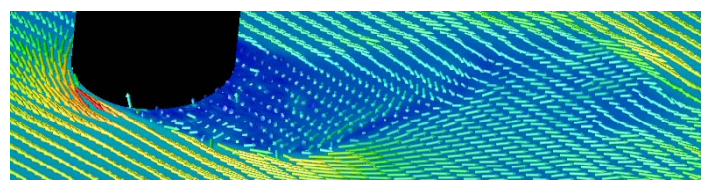

Fig. 19. Velocity vectors for cylinder at $R_{e}=10^{5}$ and $I^{*}=m^{*}$, at $\mathrm{y}=$ 2.5

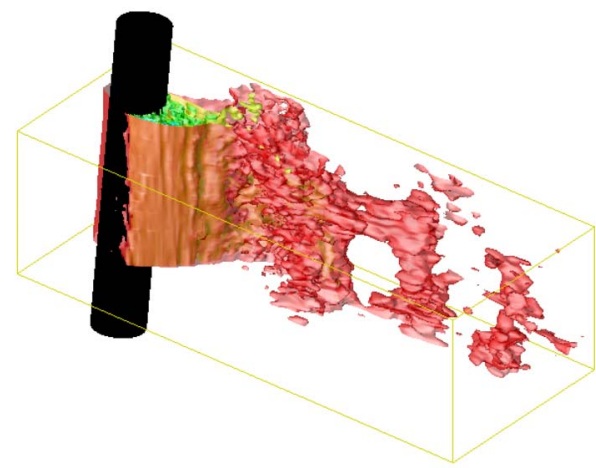

Fig. 20. Instantaneous iso-surfaces of stream-wise velocity for flow past a moving cylinder with $I^{*}=m^{*}$. Iso-surfaces $+0.5,0.2$ and $-0.5,-0.2$

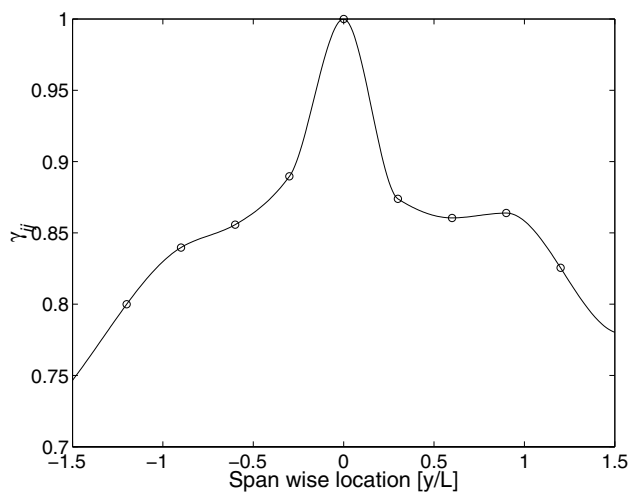

Fig. 21. Cross correlation coefficient along span of cylinder translating and rotating in cross-stream direction at $R e=10^{5}$

perfectly correlated for about eighty-five percent of the length of the cylinder. This value is only slightly lower than $\Lambda=2.7$ computed for the stationary cylinder, thus indicating that for cylinders with $I^{*}=m^{*}$ the onset of vortex induced vibration does not significantly affect the magnitude of the correlation length, although it clearly alters the flow behavior.

\section{CONCLUSIONS}

\section{A. Stationary Cylinder}

The flow around a low aspect ratio cylinder at $R e=10^{5}$ was numerically simulated using LES. One of the objectives of the simulation was to investigate the presence of threedimensional flow over the span of this cylinder, and the computational results lead to the following conclusions:

1) The simulation was assumed to be grid independent using an adaptation criterion of $c_{\text {adapt }}=4 E-05$ and the calculated values of $C_{d}, C_{l}$ and $S t$ are consistent with those reported in the literature.

2) The flow is most correlated near the mid-span and becomes less correlated near the ends of the cylinder. Furthermore, the flow is shown to be anti-symmetric about the mid-span.

3) The correlation length for this flow was computed to be 2.7, which compares reasonably well with the empirical formulas reported in the literature at this Reynolds number.

\section{B. Moving Cylinder}

The behavior of a low aspect ratio cylinder experiencing vortex induced vibrations at $R e=10^{5}$ was numerically simulated using LES. The simulations, performed for the case of a cylinder free to translate in the cross-stream direction, and also for a cylinder free to translate and rotate in a plane parallel to the cross-stream led to the following conclusions:

1) The correlation length, $\Lambda$, for the cylinder undergoing pure translation in the cross-stream direction was computed to be 3.0. This value represents an increase in correlation length compared to the value of $\Lambda=2.7$ obtained for the stationary cylinder, and indicates that the flow over the span of the $3 m$ long cylinder becomes 
perfectly correlated at the onset of vortex induced vibrations.

2) The correlation length, $\Lambda$, for the cylinder free to rotate and translate in a plane parallel to the cross-stream direction was computed to be 2.6. This value is only slightly lower than $\Lambda=2.7$ computed for the stationary cylinder, thus indicating that for cylinders with $I^{*}=m^{*}$ the onset of vortex induced vibration does not affect the magnitude of the correlation length to a large degree.

3) The assumption of two-dimensionality is only valid for the case of a cylinder undergoing pure translation. Models that restrict the motion to simple translation may govern the flow regime and not accurately reflect the behavior of an object under less rigid constraints. This has implications for current numerical and experimental techniques since these models generally only allow for cross stream and/or stream-wise motion. The extent to which the experimental apparatus or harmonic model may influence the behavior of the riser by eliminating span-wise amplitude variation is important information that should be considered for future riser designs.

\section{ACKNOWLEDGMENT}

The authors would like to thank Memorial University of Newfoundland and Dalhousie University for providing the numerical facilities required for this project. Furthermore, the financial support provided by the Natural Sciences and Engineering Research Council (NSERC) of Canada and Petroleum Research Atlantic Canada (PRAC) are gratefully acknowledged.

\section{REFERENCES}

[1] J. J. Wilde and R. H. M. Huijsmans, "Experiments for high reynolds numbers viv on risers," in The Proceedings of the Eleventh International And Polar Engineering Conference Vol. 3, 2001, pp. 400-405.

[2] M. Zdravkovich, Flow Around Circular Cylinders. New York, USA: Oxford University Press, 1997.

[3] M. Wang, P. Catalano, and G. Iaccarino, "Prediction of high reynolds number flow over a circular cylinder using les with wall modeling," Annual Research Briefs, Center for Turbulence Research, NASA Ames/Stanford Univ., pp. 351-368, 2001.

[4] K. A. Warshauer and J. A. Leene, "Experiments on mean and fluctuating pressures of circular cylinders at cross flow at very high reynolds numbers," in Proceedings Conference Wind Effects on Buildings and Structures, 1971, pp. 305-15.

[5] W. C. L. Shih, C. Wang, D. Cols, and A. Roshko, "1993 experiments on flow past rough circular cylinders at large reynolds numbers," $J$. Wind Eng. and Industrial Aerodynamics, vol. 49, pp. 351-368, 1993.

[6] E. Achenbach, "Distribution of local pressure and skin friction around a circular riser in crossflow up to re $=5,000,000$," Journal of Fluid Mechanics, vol. 34, pp. 625-639, 1968.

[7] R. Gabbai and H. Benaroya, "An overview of modeling and experiments of vortex-induced vibration of circular cylinders," Journal of Sound and Vibration, vol. 282, pp. 575-616, 2005.

[8] V. Michelassi, J. Wissink, J. Frölich, and W. Rodi, "Large-eddy simulation of flow around low-pressure turbine blades with incoming wakes," AIAA Journal, vol. 41, pp. 2143-2156, 2003.

[9] M. Tutor and A. Holdo, "Large eddy simulation of a smooth cylinder oscillating normal to uniform flow," Journal of Fluids Engineering, vol. 122, pp. 694-702, 2000.

[10] P. Bearman and I. Curie, "Pressure fluctuation measurements on an oscillating circular cylinder," Journal of Fluid Mechanics, vol. 91, pp. 661-667, 1979.
[11] H. Al-Jamal and C. Dalton, "Pressure fluctuation measurements on an oscillating circular cylinder," Journal of Fluids and Structures, vol. 19, pp. 73-92, 2004.

[12] R. Govardhan and C. Williamson, "Motions, forces and mode transitions in vortex-induced vibrations at low mass-damping," Journal of Fluid Mechanics, vol. 420, pp. 85-130, 2000.

[13] D. Brika and A. Laneville, "Vortex-induced vibrations of a long flexible cylinder," Journal of Fluid Mechanics, vol. 250, pp. 481-508, 1993.

[14] C. Williamson and A. Roshko, "Vortex formation in the wake of an oscillating cylinder," Journal of Fluids and Structures, vol. 2, pp. 355381, 1988.

[15] A. Khalak and C. Williamson, "Motions, forces and mode transitions in vortex-induced vibrations at low mass-damping," Journal of Fluids and Structures, vol. 13, pp. 813-851, 1999.

[16] R. So, Y. Liu, C. Cui, and X. Wang, "Three-dimensional wake effects on flow-induced forces," Journal of Fluids and Structures, vol. 20, pp. 373-402, 2005.

[17] F. Flemming and C. H. K. Williamson, "Vortex-induced vibrations of a pivoted cylinder," Journal of Fluid Mechanics, vol. 522, pp. 215-252, 2005.

[18] T. Bell, "The numerical wind tunnel: A three-dimensional computational fluid dynamics tool," Master's thesis, Department of Mechanical Engineering. Dalhousie University, Halifax, NS, Canada, 2003.

[19] J. Hicken, "Turbulence modeling on an unstructured time-adaptive mesh," Master's thesis, Department of Mechanical Engineering. Dalhousie University, Halifax, NS, Canada, 2004.

[20] B. R. Munson, D. F. Young, and T. H. Okiishi, Fundamentals of Fluid Mechanics: 3rd ed. New York, USA: John Wiley and Sons Inc, 1994.

[21] H. Chen, C. Chen, R. Mercier, and J. P. Pontaza, "Cfd simulation of riser viv," Deepwater Riser VIV Project Comprehensive Status Report, 2005.

[22] P. Anagnostopoulos and G. Iliadis, "Numerical study of the blockage effects on viscous flow past a circular cylinder," International Journal for Numerical Methods in Fluids, vol. 22, pp. 1061-1074, 1996.

[23] C. Norberg, "Flow around a circular cylinder: Aspects of fluctuating lift," J. Fluids and Strucures, vol. 15, pp. 459-469, 2001.

[24] G. Schewe, "On the force fluctuations acting on a circular cylinder in crossflow from subcritical up to transcritical reynolds numbers," Journal of Fluid Mechanics, vol. 133, pp. 265-285, 1983.

[25] C. Norberg, "Fluctuating lift on a circular cylinder: review and new measurements," Journal of Fluids and Structures, vol. 17, pp. 57-96, 2003.

[26] P. Bearman, "Vortex shedding from oscillating bluff bodies," Annual Review of Fluid Mechanics, vol. 16, pp. 195-222, 1984. 